\title{
Classificações e características nutricionais e tecnológicas de farinhas de mandioca comercializadas em Belém, Pará, Brasil
}

\section{Classification, nutritional and technological characteristics of cassava flour marketed in Belém, Pará, Brazil}

\author{
Elisa Cristina Andrade Neves ${ }^{1,2 *}$ (D), Gustavo Costa Nascimento ${ }^{1}$, Amanda Rios Ferreira ${ }^{1}$, \\ Daniela Andrade Neves ${ }^{1}$, André Rinaldi Fukushima ${ }^{3}$, Luís Antônio Baffile Leoni ${ }^{4}$, \\ Maria Teresa Pedrosa Silva Clerici ${ }^{1}$ (i) \\ ${ }^{1}$ Universidade Estadual de Campinas (UNICAMP), Faculdade de Engenharia de Alimentos, Departamento de \\ Tecnologia de Alimentos, Campinas/SP - Brasil \\ ${ }^{2}$ Universidade Federal do Pará (UFPA), Faculdade de Engenharia de Alimentos, Instituto de Tecnologia, Belém/PA \\ - Brasil \\ ${ }^{3}$ Faculdade de Ciências da Saúde (FASIG), Hospital IGESP, São Paulo/SP - Brasil \\ ${ }^{4}$ Centro Universitário FAM, São Paulo/SP - Brasil
}

${ }^{*}$ Corresponding Author: Elisa Cristina Andrade Neves, Universidade Estadual de Campinas (UNICAMP), Faculdade de Engenharia de Alimentos, Departamento de Tecnologia de Alimentos, Laboratório de Cereais, Raízes e Tubérculos, Rua Monteiro Lobato, 80, Cidade Universitária Zeferino Vaz, CEP: 13083-862, Campinas/SP, Brasil. E-mail: eneves@ufpa.br

Cite as: Neves, E. C. A., Nascimento, G. C., Ferreira, A. R., Neves, D. A., Fukushima, A. R., Leoni, L. A. B., Clerici, M. T. P. S. (2020). Classification, nutritional and technological characteristics of cassava flour marketed in Belém, Pará, Brazil. Brazilian Journal of Food Technology, 23, e2019143. https://doi.org/10.1590/1981-6723.14319

\begin{abstract}
Resumo
A região Norte é grande produtora de mandioca, cuja farinha tem sido consumida como parte diária do hábito alimentar da população, sendo produzida artesanalmente. Devido à escassez de informaç̃̃es técnicas, este trabalho objetivou classificar e caracterizar nutricional e tecnologicamente nove amostras de farinhas de mandioca adquiridas em Belém-PA. Os resultados foram submetidos à análise de variância e, quando significativos $(p<0,05)$, foi feito o teste de diferença de médias Scott Knott. As farinhas estavam dentro dos padrões da legislação brasileira em relação a umidade e cinzas, e foram classificadas como de alta acidez, sendo que quatro das nove farinhas apresentaram teor de cianetos entre 15 e $31 \mathrm{mg} \mathrm{HCN} / \mathrm{kg}$. Apesar de serem seguras para o consumo, recomendam-se melhorias em etapas de processo para diminuir o teor de cianetos. Quanto a cor e granulometria, foram consideradas amarelas (67\%) e brancas (33\%) e grossas (67\%) e médias (33\%), respectivamente. Nutricionalmente, podem contribuir como fonte de fibra alimentar $(7,67 \%$ a $11,26 \%)$ e amido cru, sendo benéficas para dar saciedade. Tecnologicamente, apresentaram-se como espessantes, por apresentarem amido cru, tanto no consumo direto quanto em produtos cozidos, formando pastas claras, textura longa e não firmes. Estas variações nas características nutricionais e tecnológicas permitiram verificar que são necessárias modificações no processamento para padronização da qualidade das farinhas utilizadas como ingrediente alimentício sem glúten.
\end{abstract}

Palavras-chave: Manihot esculenta; Propriedades físico-químicas; Compostos tóxicos; Carboidratos; Propriedades de pasta; Força do gel. 


\begin{abstract}
The northern region of Brazil is a large cassava producer, and cassava flour has been handcrafted and consumed daily by the local population. This study aimed to classify and characterize nine different samples of cassava flour marketed in Belém-PA/ Brazil from a nutritional and technological point of view. The results were subjected to analysis of variance and the averages were compared using the Scott Knott test $(p<0.05)$. The flours were within the legislation in relation to moisture and ash contents, with high acidity, four out of nine flours evaluated had cyanide content between 15 and $31 \mathrm{mg} \mathrm{HCN} / \mathrm{kg}$. Regarding color and particle size, samples varied between yellow (67\%) and white (33\%); from coarse $(67 \%)$ to medium $(33 \%)$, respectively. From a nutritional point of view, the cassava flours are a source of dietary fiber $(7.67 \%$ to $11.26 \%)$ and raw starch, which have satiety benefits. From the technological point of view, the flours are used as thickeners, producing pastes that are clear with a long and less firm texture. These variations in nutritional and technological characteristics allowed to verify that changes in processing are necessary to standardize the quality of flours used as a gluten free food ingredient.
\end{abstract}

Keywords: Manihot esculenta; Physicochemical properties; Toxic compounds; Carbohydrates; Paste properties; Gel strength.

\title{
1 Introdução
}

As raízes tuberosas de mandioca (Manihot esculenta Crantz) são amiláceas e estão entre os alimentos fundamentais na segurança alimentar da população brasileira. Segundo o Instituto Brasileiro de Geografia Estatística (Instituto Brasileiro de Geografia e Estatística, 2019), a produção de mandioca no Brasil praticamente se estabilizou na média de 22 milhões de toneladas/ano nas últimas safras. A região Norte do Brasil é responsável por 33\% dessa produção, com destaque para o Pará, com 19\% da produção brasileira.

A mandioca deve ser imediatamente consumida cozida, congelada ou submetida a processos térmicos de secagem, para evitar perdas pós-colheita, pois apresentam teor de umidade superior a $60 \%$, escurecimento enzimático e rápida deterioração fisiológica e microbiana. $\mathrm{O}$ tratamento térmico será sempre importante na obtenção de derivados de mandioca, pois, além de inativar o sistema enzimático, favorece a eliminação dos compostos cianogênicos presentes na mandioca in natura, diminuindo assim a sua toxidez (Franck et al., 2011), sendo que parte dos compostos tóxicos também pode ser eliminada por lixiviação durante a prensagem.

Na região Norte do Brasil, o derivado de mandioca com maior produção é a farinha, a qual é produzida em unidades conhecidas como casas de farinhas, apresentando tal atividade grande importância socioeconômica, visto que é fonte de renda para a agricultura familiar. Consumida por grande parte da população durante as principais refeições, sendo que, muitas vezes, os consumidores são fiéis em relação à sua aquisição em determinados locais de produção ou ao ponto de venda, pois relacionam suas características à origem.

Para produção da farinha, utiliza-se a mandioca, branca ou amarela, de maior teor de compostos cianogênicos, pois esta não é indicada para consumo como mandioca de mesa. O processo pode ser artesanal ou semi-industrial, e apresenta as etapas de descascamento, lavagem, trituração, prensagem, esfarelamento e gelatinização parcial, com formação de aglomerados, que interferem na granulometria do produto (Bezerra, 2006; Cereda \& Vilpoux, 2003); posteriormente, realizam-se a torração, com temperaturas variando de 150 a $250{ }^{\circ} \mathrm{C}$, e o peneiramento (Brito et al., 2015), para ajuste da granulometria desejada.

Este trabalho teve como objetivo caracterizar nove farinhas de mandioca, comercializadas no complexo do mercado Ver-o-Peso, em Belém-PA, Brasil, em relação às características físicas, tecnológicas e nutricionais. 


\section{Material e métodos}

\subsection{Obtenção das farinhas}

Foram adquiridas nove amostras de farinhas de mandioca (FM1 a FM9), em março de 2017, na feira livre do Complexo Ver-o-Peso, em Belém-PA (Coordenadas - $\left.01^{\circ} 27^{\prime} 21^{\prime \prime} ;-48^{\circ} 30^{\prime} 16^{\prime \prime}\right)$, todas provenientes de casas de farinhas com processo artesanal.

\subsection{Classificação das farinhas de mandioca}

Para a classificação das farinhas em relação às classes, grossa, média ou fina (Brasil, 2011), foi realizada a análise de granulometria, utilizando o método 965.22 (Association of Official Analytical Chemists, 2005), por meio de peneiras de $2 \mathrm{~mm}$ e $1 \mathrm{~mm}$ de aberturas. A distribuição granulométrica foi avaliada utilizando-se uma série de sete peneiras com aberturas entre $2 \mathrm{~mm}$ e $0,25 \mathrm{~mm}$.

O volume específico (VE) foi calculado pela relação entre volume e massa da amostra, e expresso em mL/g.

Os parâmetros de cor foram obtidos no sistema CIELab ( $\mathrm{L}^{*}, \mathrm{a}^{*}$ e $\mathrm{b}^{*}$ ), usando um colorímetro CR-10 (Konica Minolta, Tóquio, Japão), com iluminante D65. As farinhas foram classificadas em relação à cor em branca, amarela ou creme (Brasil, 2011).

Para as demais análises, as farinhas foram trituradas e peneiradas, sendo a granulometria padronizada para 60 mesh.

Para determinação do teor de compostos glicosídeos cianogênicos totais, expresso em teor de cianeto total, utilizou-se a metodologia de Oliveira \& Oliveira (2010), conhecida como método Konigi. No processo de validação, este método teve um limite de detecção inferior de 5 ppm e um limite inferior de quantificação de 10 ppm de cianeto.

$\mathrm{O} \mathrm{pH}$ e a acidez titulável foram determinados segundo Association of Official Analytical Chemists (2005). As farinhas foram classificadas, considerando a legislação brasileira, em acidez baixa $(<3$ meq $\mathrm{NaOH}$ $0,1 \mathrm{~N} / 100 \mathrm{~g}$ ) ou acidez alta (> 3 meq NaOH 0,1N/100 g) (Brasil, 2011).

A atividade de água (Aw) foi determinada em higrômetro à temperatura ambiente $\left(25^{\circ} \mathrm{C}\right)$.

\subsection{Caracterização nutricional das farinhas de mandioca}

Para se estabelecer a composição em nutrientes das farinhas de mandioca, foram realizadas as seguintes análises em triplicata, de acordo com os métodos oficiais da Association of Official Analytical Chemists (2005): teor de umidade (método 925.09); teor de proteínas (método 920.87); extrato etéreo (método 920.85); cinzas (método 923.03), e fibras alimentares totais (método 985-29). Os teores de carboidratos digeríveis foram calculados por diferença e o valor energético foi obtido considerando os fatores de conversão de energia: $9 \mathrm{kcal} / \mathrm{g}$ para lipídios, $4 \mathrm{kcal} / \mathrm{g}$ para proteínas e $4 \mathrm{kcal} / \mathrm{g}$ para carboidratos digeríveis.

\subsection{Caracterização tecnológica das farinhas de mandioca}

O índice de absorção de água (IAA) e o índice de solubilidade em água (ISA) foram determinados conforme metodologia de Anderson et al. (1969).

As propriedades de pasta foram avaliadas em Rapid Visco-Analyser (RVA), modelo RVA 4500, utilizando como parâmetro standard 1, software Thermocline).

A pasta formada durante a análise das farinhas de mandioca no RVA foi distribuída em dois porta-amostras, resfriada e mantida sob refrigeração por 24 horas. A força do gel foi determinada utilizando Texturômetro Stable Microssystems Texture Analyser TAXT2 (Surrey, Inglaterra), com sonda cilíndrica de acrílico 10 Dia Cylinder Derrm $(\mathrm{P} / 10)$. 


\subsection{Análise estatística dos resultados}

As análises foram realizadas em triplicata, com exceção do teor de cianeto livre e da granulometria, e os resultados foram submetidos à análise de variância (ANOVA) com o uso do sistema SISVAR, versão 5.6 (Universidade Federal de Lavras, Lavras, MG, Brasil), no nível de significância de 5\%. Quando significativos, foi utilizado o teste Scott Knott para determinar as diferenças estatísticas entre as médias $(p<0,05)$ (Ferreira, 2008).

\section{Resultados e discussão}

\subsection{Classificação das farinhas de mandioca}

Considerando os padrões estabelecidos pela legislação brasileira (Brasil, 2011) em relação à granulometria, verificou-se, na Tabela 1 e na Figura 1, que a maioria (67\%) das farinhas de mandioca foi classificada como grossa (mais que $10 \%$ da massa retida na peneira de abertura de $2 \mathrm{~mm}$ ) e $33 \%$ como média.
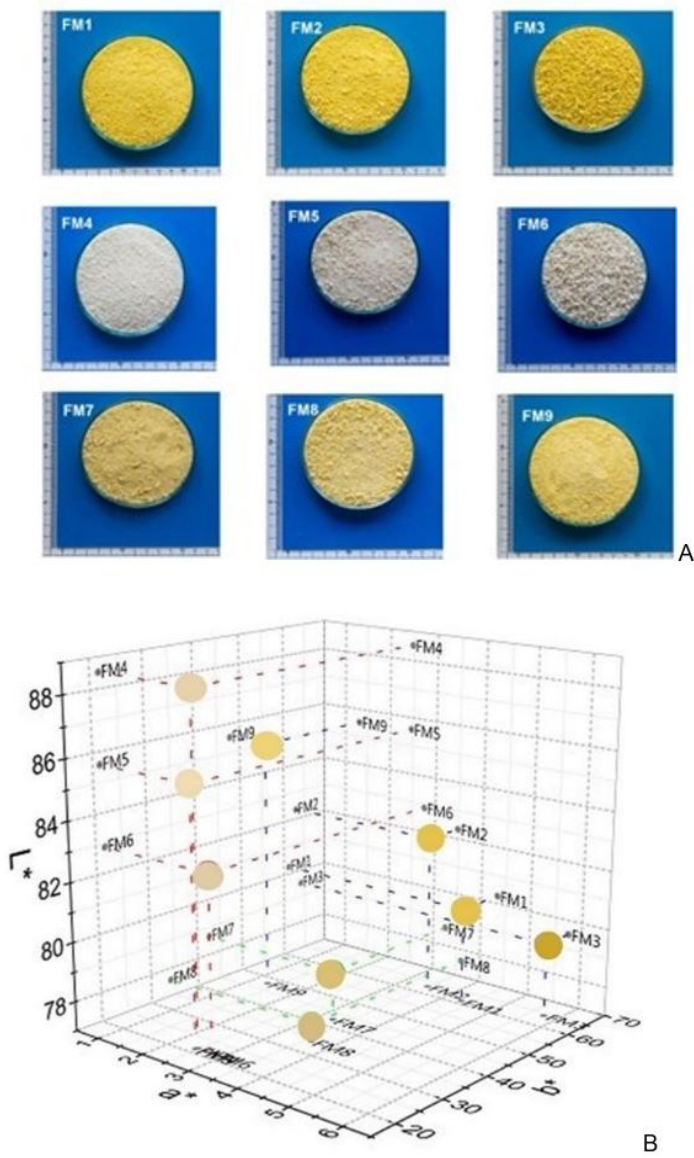

Figura 1. Imagens das farinhas de mandioca, considerando cor e granulometria originais (A) e parâmetros de cor $\left(\mathrm{L}^{*}, \mathrm{a}^{*} \mathrm{e} \mathrm{b}^{*}\right)$ no sistema CIELab (B).

Pela Tabela 1 e Figura 2, a FM6 pode ser considerada a farinha mais homogênea, sendo que a massa retida foi principalmente na peneira de abertura $2 \mathrm{~mm}$, apresentado o maior valor de granulometria e volume específico. As FM1, FM2, FM5 e FM9 apresentaram-se heterogêneas, com massa retida distribuída em todas as peneiras, enquanto, as demais farinhas estavam distribuídas mais nas peneiras de maiores aberturas (acima de $1,19 \mathrm{~mm})$. 
Tabela 1. Classificação quanto a granulometria, volume específico, teores de cianeto total, pH, acidez, parâmetros de cor e atividade de água das farinhas de mandioca (FM) comercializadas em Belém-PA ${ }^{\mathrm{a}}$.

\begin{tabular}{|c|c|c|c|c|c|c|c|c|c|c|c|c|c|}
\hline \multirow[b]{2}{*}{ Farinhas } & \multicolumn{3}{|c|}{ Granulometria } & \multirow[b]{2}{*}{$\begin{array}{c}\text { Volume } \\
\text { específico } \\
(\mathrm{mL} / \mathrm{g})\end{array}$} & \multirow[b]{2}{*}{$\begin{array}{r}\text { Cianeto total } \\
(\mathrm{mg} \mathrm{HCN} / \mathrm{kg})\end{array}$} & \multirow[b]{2}{*}{$\mathbf{p H}$} & \multicolumn{2}{|c|}{ Acidez } & \multicolumn{4}{|c|}{ Parâmetros de cor ${ }^{d}$} & \multirow[b]{2}{*}{$\begin{array}{c}\text { Atividade de } \\
\text { água }\end{array}$} \\
\hline & $\begin{array}{l}\text { Retenção (\%) } \\
(2,0 \mathrm{~mm})\end{array}$ & $\begin{array}{c}\text { Retenção } \\
(\%) \\
(1,0 \text { mm) }\end{array}$ & Classe $^{b}$ & & & & $\begin{array}{c}\text { Acidez } \\
\text { (meq NaOH } \\
\mathbf{0 , 1} \mathbf{N} / 100 \mathrm{~g})\end{array}$ & Classe $^{\mathrm{c}}$ & $\mathbf{L}^{*}$ & $a^{*}$ & $\mathbf{b}^{*}$ & Classe & \\
\hline FM1 & 0,64 & 21,07 & Média & $1,48 \pm 0,02^{\mathrm{b}}$ & 15,50 & $4,37 \pm 0,02^{\mathrm{e}}$ & $6,63 \pm 0,63^{\mathrm{b}}$ & Alta & $80,27 \pm 0,5^{\mathrm{d}}$ & $4,33 \pm 0,16^{\mathrm{b}}$ & $61,15 \pm 0,77^{\mathrm{c}}$ & Amarela & $0,45 \pm 0,01^{\mathrm{f}}$ \\
\hline FM2 & 16,07 & 21,20 & Grossa & $1,49 \pm 0,01^{\mathrm{b}}$ & $<5,0$ & $4,27 \pm 0,01^{\mathrm{f}}$ & $6,85 \pm 0,06^{\mathrm{b}}$ & Alta & $82,34 \pm 0,11^{\mathrm{c}}$ & $3,48 \pm 0,03^{\mathrm{c}}$ & $62,91 \pm 0,36^{b}$ & Amarela & $0,52 \pm 0,01^{\mathrm{a}}$ \\
\hline FM3 & 46,94 & 26,60 & Grossa & $1,48 \pm 0,00^{\mathrm{b}}$ & 30,53 & $4,14 \pm 0,02^{\mathrm{g}}$ & $7,39 \pm 0,16^{\mathrm{a}}$ & Alta & $79,51 \pm 0,19^{d}$ & $5,75 \pm 0,19^{\mathrm{a}}$ & $64,11 \pm 0,71^{\mathrm{a}}$ & Amarela & $0,47 \pm 0,01^{\mathrm{e}}$ \\
\hline FM4 & 0,51 & 7,00 & Média & $1,44 \pm 0,01^{\mathrm{b}}$ & $<5,0$ & $4,29 \pm 0,00^{\mathrm{c}}$ & $6,48 \pm 0,20^{\mathrm{b}}$ & Alta & $88,52 \pm 0,37^{\mathrm{a}}$ & $2,47 \pm 0,05^{\mathrm{d}}$ & $21,66 \pm 0,19^{g}$ & Branca & $0,49 \pm 0,01^{\mathrm{c}}$ \\
\hline FM5 & 15,21 & 21,61 & Grossa & $1,53 \pm 0,01^{\mathrm{b}}$ & $<5,0$ & $4,57 \pm 0,02^{\mathrm{c}}$ & $5,47 \pm 0,14^{\mathrm{c}}$ & Alta & $85,58 \pm 0,24^{b}$ & $2,46 \pm 0,01^{\mathrm{d}}$ & $21,31 \pm 0,12^{\mathrm{g}}$ & Branca & $0,48 \pm 0,00^{\mathrm{d}}$ \\
\hline FM6 & 96,36 & 3,42 & Grossa & $1,69 \pm 0,01^{\mathrm{a}}$ & 17,10 & $4,42 \pm 0,00^{\mathrm{d}}$ & $4,95 \pm 0,11^{\mathrm{d}}$ & Alta & $82,86 \pm 0,29^{\mathrm{c}}$ & $2,76 \pm 0,17^{\mathrm{d}}$ & $21,72 \pm 0,27^{\mathrm{g}}$ & Branca & $0,51 \pm 0,00^{\mathrm{b}}$ \\
\hline FM7 & 45,06 & 27,91 & Grossa & $1,3 \pm 0,00^{\mathrm{b}}$ & $<5,0$ & $4,68 \pm 0,02^{\mathrm{b}}$ & $4,25 \pm 0,19^{\mathrm{e}}$ & Alta & $78,66 \pm 0,39^{\mathrm{e}}$ & $3,26 \pm 0,16^{\mathrm{c}}$ & $42,54 \pm 0,60^{\mathrm{e}}$ & Amarela & $0,36 \pm 0,01^{\mathrm{g}}$ \\
\hline FM8 & 45,06 & 25,54 & Grossa & $1,33 \pm 0,01^{\mathrm{b}}$ & $<5,0$ & $5,25 \pm 0,00^{\mathrm{a}}$ & $5,55 \pm 0,09^{\mathrm{c}}$ & Alta & $77,58 \pm 0,45^{\mathrm{f}}$ & $3,61 \pm 0,22^{\mathrm{c}}$ & $34,09 \pm 0,22^{\mathrm{f}}$ & Amarela & $0,36 \pm 0,01^{\mathrm{g}}$ \\
\hline FM9 & 0,75 & 21,17 & Média & $1,53 \pm 0,02^{b}$ & 20,23 & $4,23 \pm 0,02^{\mathrm{c}}$ & $5,93 \pm 0,35^{\mathrm{c}}$ & Alta & $85,53 \pm 0,61^{b}$ & $1,31 \pm 0,24^{\mathrm{e}}$ & $47,8 \pm 0,65^{\mathrm{d}}$ & Amarela & $0,48 \pm 0,01^{\mathrm{d}}$ \\
\hline
\end{tabular}

${ }^{a}$ Médias + desvio padrão. Letras minúsculas diferentes na mesma coluna diferem entre si pelo teste de Scott-Knott $(p<0,05) ;{ }^{b}$ Classificação considerando a granulometria baseada na legislação (Brasil, 2011); ${ }^{\mathrm{c}}$ Baixa acidez ( $<3$ meq NaOH $0,1 \mathrm{~N} / 100 \mathrm{~g}) ;$ alta acidez $\left(>3\right.$ meq NaOH $0,1 \mathrm{~N} / 100 \mathrm{~g}$ (Brasil, 2011); ${ }^{\mathrm{d}} \mathrm{L}^{*}=$ luminosidade; $\mathrm{a}^{*}=$ coordenada vermelho/verde $\left(+\mathrm{a}\right.$ indica vermelho e $-\mathrm{a}$ indica verde); $\mathrm{b}^{*}=$ coordenada amarelo/azul ( $+\mathrm{b}$ indica amarelo $\mathrm{e}-\mathrm{b}$ indica azul). 


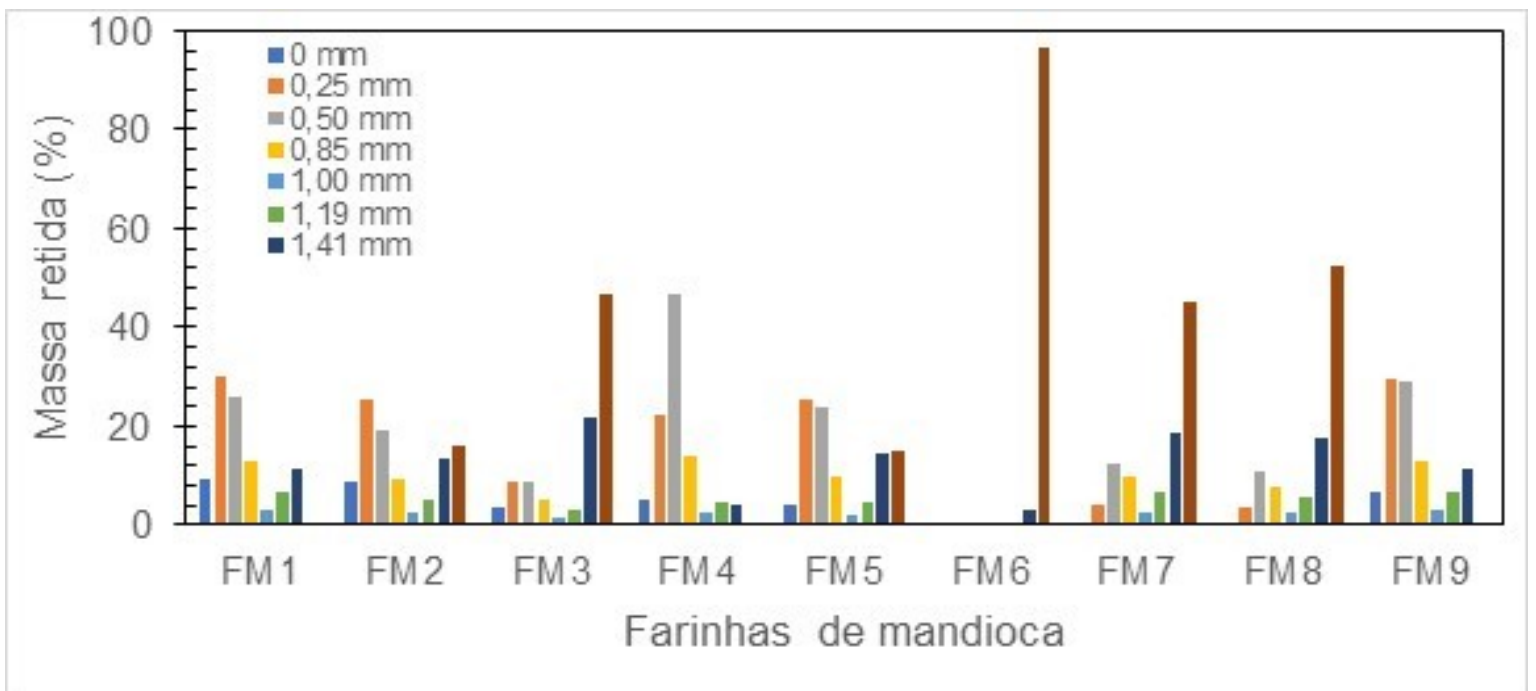

Figura 2. Distribuição granulométrica das farinhas de mandioca.

Esta diferença de granulometria entre as farinhas foi explicada por Brito et al. (2015), uma vez que, durante o processamento artesanal de farinha de mandioca, principalmente durante as etapas de pré-gelatinização e torração, ocorre formação heterogênea de aglomerados devido à gelatinização parcial do amido e à agregação das fibras presentes na massa de mandioca ralada. Este parâmetro depende do tipo e da carga dos fornos, temperaturas e tempos utilizados em cada etapa do processo e do posterior peneiramento com diferentes aberturas.

Verificou-se, na Tabela 1, que 55,6\% das farinhas apresentaram teores de cianetos totais menores que $5 \mathrm{mg} \mathrm{HCN} / \mathrm{kg}$ e 44,4\%, com teores entre 15 e $31 \mathrm{mg} \mathrm{HCN} / \mathrm{kg}$, sendo seguras para consumo, pois são menores que os teores que poderiam ocasionar intoxicação. Observe-se que Organização Mundial da Saúde estipulou que a dose letal para ingestão de compostos cianogênicos é de $10 \mathrm{mg} / \mathrm{kg}$ de peso (Chisté et al., 2010).

Segundo Brimer \& Rosling (1993), quantidades de HCN ingeridas são lentamente eliminadas pelo organismo dos animais de estômago ácido, incluindo os humanos. Neste mecanismo de eliminação, é envolvido o aminoácido cisteína, o que explica que dietas ricas em proteínas ajudam o organismo no processo de desintoxicação. Consequentemente, como a farinha de mandioca é associada nas refeições principais com carnes, frangos e peixes, o risco de intoxicação é baixo.

Cohen et al. (2007) observaram teores de compostos cianogênicos entre 7,75 e 20,60 mg HCN/kg em farinhas secas produzidas no Pará, Brasil. Aryee et al. (2006) encontraram teores entre 58 e $200 \mathrm{mg} \mathrm{HCN} / \mathrm{kg}$ para farinhas secas ao sol, e Eleazu et al. (2011), entre 8,14 e 12,72 $\mathrm{mg} \mathrm{HCN} / \mathrm{kg}$ para farinhas secas, produzidas na Nigéria.

As variações nos teores de cianeto obtidas neste trabalho e nos resultados encontrados na literatura podem ser explicadas por Cereda \& Vilpoux (2003) e por Chisté et al. (2010), pois os teores determinados nas farinhas de mandioca analisadas podem indicar que as etapas do processamento, como trituração, prensagem, prégelatinização e torração, foram eficientes para diminuição do princípio tóxico, seja por evaporação ou lixiviação. Porém, os teores de cianeto dependem também dos valores iniciais deste composto, que podem variar de acordo com a espécie de mandioca e as condições ambientais.

Observou-se que todas as farinhas analisadas apresentaram acidez alta, segundo os padrões da legislação brasileira (> 3,0 meq. NaOH 0,1 N/100 g) (Brasil, 2011). Outros autores, Silva et al. (2015); Álvares et al. (2016) e Chisté et al. (2006), também obtiveram resultados semelhantes aos obtidos neste trabalho e apresentaram a explicação de que, durante o tempo de prensagem da massa de mandioca triturada, pode ocorrer maior ou menor grau de fermentação, o que influencia nos teores de acidez. 
Pela Figura 1 e Tabela 1, verificou-se que o parâmetro b* próximo a 21 e entre 34 e 64 possibilitou classificar as farinhas em brancas ou amarelas, respectivamente. As farinhas mais escuras foram as amarelas (FM8, FM7 e FM3) e as mais claras, as brancas (FM4, FM5 e FM6), segundo o parâmetro L*. O parâmetro $\mathrm{a}^{*}$, apesar de $p<0,05$, variou pouco, de 1 a 5 .

As FM1, FM4 e FM9, que eram de granulometria média e com cores amarelas ou brancas (Figura 1, Tabela 1), são mais utilizadas em Belém-PA para elaboração de farofas e empanados sem glúten. Enquanto as demais, classificadas como grossas, são consumidas nas refeições (observações do autor, ao adquirir as farinhas).

Todas as farinhas apresentaram atividades de água inferiores a 0,6 , ou seja, abaixo do limite mínimo, o que pode possibilitar o desenvolvimento de micro-organismos (Jay, 2009).

\subsection{Caracterização nutricional das farinhas de mandioca}

As farinhas de mandioca se encontravam de acordo com a legislação brasileira (Brasil, 2011) em relação a umidade e cinzas, com teores inferiores a 13\% e 1,4\% respectivamente. Silva et al. (2015) e Dias \& Leonel (2006) encontraram variações de umidade entre as farinhas por eles analisadas, mas inferiores a 13\%, o que garante, segundo Álvares et al. (2016), estabilidade durante armazenamento.

FM4 e FM5 apresentaram os maiores valores de cinzas $(p<0,05)$. Chisté et al. (2006) observaram valores de cinzas semelhantes aos deste trabalho em farinhas de mandioca secas produzidas no Pará.

Com relação ao teor de fibras alimentares totais (Tabela 2), incluindo fibras solúveis e insolúveis, FM2 e FM5 apresentaram 11,26\% e 10,28\%, os maiores teores $(p<0,05)$, respectivamente, em relação às demais, que foram acima de $7 \%$.

Tabela 2. Caracterização nutricional das farinhas de mandioca (FM) comercializadas em Belém-PA

\begin{tabular}{cccccccc}
\hline Farinhas & $\begin{array}{c}\text { Umidade } \\
\mathbf{( \% )}\end{array}$ & $\begin{array}{c}\text { Proteínas } \\
\mathbf{( \% )}\end{array}$ & $\begin{array}{c}\text { Extrato etéreo } \\
\mathbf{( \% )}\end{array}$ & $\begin{array}{c}\text { Cinzas } \\
\mathbf{( \% )}\end{array}$ & $\begin{array}{c}\text { Fibras } \\
\text { Alimentares } \\
\text { Totais } \\
\mathbf{( \% )}\end{array}$ & $\begin{array}{c}\text { Carboidratos } \\
\text { Digeríveis } \\
\mathbf{( \% )}\end{array}$ & $\begin{array}{c}\text { Valor } \\
\text { Energético } \\
(\mathbf{k c a l} / \mathbf{g})\end{array}$ \\
\hline FM1 & $7,11 \pm 0,22^{\mathrm{b}}$ & $1,34 \pm 0,07^{\mathrm{a}}$ & $0,16 \pm 0,01^{\mathrm{c}}$ & $0,75 \pm 0,03^{\mathrm{a}}$ & $7,67 \pm 0,68^{\mathrm{b}}$ & $82,97 \pm 0,81^{\mathrm{a}}$ & $338,67 \pm 3,56^{\mathrm{a}}$ \\
\hline FM2 & $7,13 \pm 0,34^{\mathrm{b}}$ & $1,23 \pm 0,04^{\mathrm{a}}$ & $0,06 \pm 0,04^{\mathrm{c}}$ & $0,68 \pm 0,02^{\mathrm{b}}$ & $11,26 \pm 2,12^{\mathrm{a}}$ & $79,64 \pm 2,00^{\mathrm{b}}$ & $324,04 \pm 7,51^{\mathrm{b}}$ \\
\hline FM3 & $6,98 \pm 0,15^{\mathrm{b}}$ & $1,16 \pm 0,06^{\mathrm{b}}$ & $0,04 \pm 0,02^{\mathrm{c}}$ & $0,71 \pm 0,00^{\mathrm{b}}$ & $8,54 \pm 0,44^{\mathrm{b}}$ & $82,56 \pm 0,54^{\mathrm{a}}$ & $335,31 \pm 2,34^{\mathrm{a}}$ \\
\hline FM4 & $7,93 \pm 0,08^{\mathrm{a}}$ & $1,14 \pm 0,03^{\mathrm{a}}$ & $0,17 \pm 0,08^{\mathrm{c}}$ & $0,68 \pm 0,02^{\mathrm{b}}$ & $8,18 \pm 1,35^{\mathrm{b}}$ & $81,64 \pm 1,39^{\mathrm{a}}$ & $333,68 \pm 5,36^{\mathrm{a}}$ \\
\hline FM5 & $7,92 \pm 0,05^{\mathrm{a}}$ & $1,09 \pm 0,08^{\mathrm{b}}$ & $0,37 \pm 0,07^{\mathrm{b}}$ & $0,66 \pm 0,02^{\mathrm{b}}$ & $10,68 \pm 3,51^{\mathrm{a}}$ & $79,28 \pm 3,46^{\mathrm{b}}$ & $324,85 \pm 14,36^{\mathrm{b}}$ \\
\hline FM6 & $8,14 \pm 0,04^{\mathrm{a}}$ & $1,24 \pm 0,11^{\mathrm{a}}$ & $0,68 \pm 0,11^{\mathrm{a}}$ & $0,50 \pm 0,05^{\mathrm{c}}$ & $7,87 \pm 1,33^{\mathrm{b}}$ & $81,57 \pm 1,35^{\mathrm{a}}$ & $337,40 \pm 5,16^{\mathrm{a}}$ \\
\hline FM7 & $7,15 \pm 0,05^{\mathrm{b}}$ & $1,25 \pm 0,10^{\mathrm{a}}$ & $0,14 \pm 0,05^{\mathrm{c}}$ & $0,69 \pm 0,01^{\mathrm{b}}$ & $8,25 \pm 0,47^{\mathrm{b}}$ & $83,52 \pm 0,46^{\mathrm{a}}$ & $336,35 \pm 2,08^{\mathrm{a}}$ \\
\hline FM8 & $6,28 \pm 0,26^{\mathrm{c}}$ & $1,14 \pm 0,01^{\mathrm{b}}$ & $0,11 \pm 0,03^{\mathrm{c}}$ & $0,76 \pm 0,01^{\mathrm{a}}$ & $8,64 \pm 1,14^{\mathrm{b}}$ & $83,08 \pm 0,98^{\mathrm{a}}$ & $337,84 \pm 3,72^{\mathrm{a}}$ \\
\hline FM9 & $6,27 \pm 0,12^{\mathrm{c}}$ & $1,02 \pm 0,13^{\mathrm{b}}$ & $0,50 \pm 0,25^{\mathrm{b}}$ & $0,66 \pm 0,02^{\mathrm{b}}$ & $7,76 \pm 0,28^{\mathrm{b}}$ & $83,79 \pm 0,71^{\mathrm{a}}$ & $343,79 \pm 0,94^{\mathrm{a}}$ \\
\hline
\end{tabular}

${ }^{a}$ Médias \pm desvio padrão. Letras minúsculas diferentes na mesma coluna diferem entre si pelo teste de $\operatorname{Scott-Knott~}(p<0,05)$.

Os teores de fibras alimentares encontrados no presente trabalho foram superiores ao apresentado na Tabela Brasileira de Composição de Alimentos (2011) para farinha de mandioca torrada, que foi 6,5\%, refletindo as diferenças entre as farinhas analisadas. A farinha de mandioca é consumida na refeição principal, quando substitui parcialmente o arroz (1,6\% de fibra alimentar (Tabela Brasileira de Composição de Alimentos, 2011), portanto poderá contribuir com maior ingestão de fibras, favorecendo a digestão lenta do amido e aumentando assim a saciedade.

A legislação brasileira não estabelece padrões para teores de proteínas e extrato etéreo, devido ao fato dos valores serem inferiores a $2 \%$, apesar de diferirem entre si, todas as farinhas apresentaram teores de proteínas e de extrato etéreo inferior a $1,5 \%$ e $1 \%$, respectivamente. 
O valor energético das farinhas de mandioca pode variar de acordo com o consumo, pois se estas farinhas forem cozidas, tendo água disponível, como em caldos, sopas e pirão, o amido pode ficar completamente gelatinizado e fornecer o valor energético próximo ao calculado neste trabalho; porém, se for usada, como a maioria dos casos, como espessante e adicionada no momento do consumo, como em caldos de feijão e tigelas de açaí, o amido poderá estar cru e ser de difícil acesso pelas enzimas digestivas.

Esta digestão lenta foi bem explicada no trabalho de Brito et al. (2015), que avaliaram a digestibilidade em relação aos carboidratos digeríveis de farinhas de mandioca, de acordo com o consumo popular no Brasil, em que é usada como espessante, sem passar por cozimento, pois usaram a temperatura de $37{ }^{\circ} \mathrm{C}$ durante toda a análise. Os autores verificaram diferenças entre os produtos, mostrando que a presença de amido cru e dos açúcares pode variar de acordo com as características físico-químicas e de processamento, incluindo o fato de que algumas apresentaram digestão mais lenta e menor valor energético que outras, no período analisado de duas horas.

\subsection{Caracterização tecnológica das farinhas de mandioca}

Os parâmetros tecnológicos podem orientar o consumidor para os diferentes usos de uma farinha. Por exemplo, farinhas com maior absorção de água, como as FM3 e FM7, podem ser utilizadas como espessantes em alimentos para pessoas com disfagias, em que pequenas concentrações podem ser usadas e, assim, evitar um aumento energético elevado do produto espessado, quando se faz uso de amidos com elevado IAA. Quando uma farinha apresenta alta solubilidade em água, ela pode ser utilizada para aumentar os sólidos solúveis em bebidas e caldos, e ser fonte imediata de glicose, pois o amido presente pode estar dextrinizado, logo pode ser um substituto natural para produtos que necessitam do uso de maltodextrinas para seu processamento.

Verificou-se, na Tabela 3, que FM3 e FM6 apresentaram os maiores índices de absorção de água (IAA). $\mathrm{Na}$ farinha FM4, seguida da FM5, foram observados os maiores valores de índice de solubilidade em água (ISA), o que pode indicar maior disponibilidade de grupos hidrofilicos $(-\mathrm{OH})$ para se ligarem às moléculas de água e uma maior capacidade de formação de gel das moléculas de amido.

Tabela 3. Índice de absorção de água (IAA), índice de solubilidade em água (ISA), propriedades de pasta e força do gel de farinhas de mandioca $(\mathrm{FM})^{\mathrm{a}}$.

\begin{tabular}{|c|c|c|c|c|c|c|c|c|c|c|}
\hline \multirow[b]{2}{*}{$\begin{array}{c}\text { Farinhas de } \\
\text { mandioca }\end{array}$} & \multirow[b]{2}{*}{ IAA } & \multirow[b]{2}{*}{ ISA } & \multicolumn{7}{|c|}{ Propriedades de pasta $^{b}$} & \multirow[b]{2}{*}{$\begin{array}{c}\text { Força } \\
\text { do gel } \\
(\mathbf{N}) \\
\end{array}$} \\
\hline & & & $\begin{array}{l}\mathbf{T p} \\
\left({ }^{\circ} \mathbf{C}\right)\end{array}$ & $\begin{array}{c}\text { VM } \\
\text { a } 95^{\circ} \mathrm{C} \\
\text { (cP) }\end{array}$ & $\begin{array}{c}\mathbf{t}_{\text {pico }} \\
(\mathrm{min})\end{array}$ & $\begin{array}{c}\mathrm{Vm} \\
\text { a } 95^{\circ} \mathrm{C} \\
(\mathrm{cP})\end{array}$ & $\begin{array}{c}\mathrm{Q} \\
(\mathrm{cP})\end{array}$ & 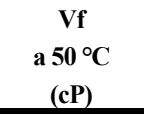 & $\begin{array}{c}\mathrm{R} \\
\text { (cP) }\end{array}$ & \\
\hline FM1 & $6,42 \pm 0,10^{b}$ & $12,57 \pm 0,26^{\mathrm{c}}$ & $76,70 \pm 0,09^{\text {ns }}$ & $2140 \pm 32^{\mathrm{d}}$ & $5,60 \pm 0,09^{\mathrm{b}}$ & $1594 \pm 17^{\mathrm{b}}$ & $547 \pm 16^{\mathrm{a}}$ & $2404 \pm 8,5^{\mathrm{e}}$ & $810 \pm 8^{c}$ & $0,37 \pm 0,03^{b}$ \\
\hline FM2 & $6,23 \pm 0,02^{\mathrm{b}}$ & $7,88 \pm 0,16^{\mathrm{e}}$ & $77,40 \pm 0,07^{\mathrm{ns}}$ & $2091 \pm 9^{d}$ & $5,73 \pm 0,09^{\mathrm{b}}$ & $1670 \pm 20^{\mathrm{b}}$ & $421 \pm 11^{\mathrm{b}}$ & $2516 \pm 20^{\mathrm{d}}$ & $846 \pm 1^{\mathrm{c}}$ & $0,38 \pm 0,02^{\mathrm{b}}$ \\
\hline FM3 & $7,18 \pm 0,13^{\mathrm{a}}$ & $9,10 \pm 0,35^{\mathrm{d}}$ & $79,80 \pm 0,04^{\mathrm{ns}}$ & $2142 \pm 36^{\mathrm{d}}$ & $6,37 \pm 0,24^{\mathrm{a}}$ & $1964 \pm 62^{\mathrm{a}}$ & $178 \pm 26^{\mathrm{d}}$ & $2964 \pm 77,5^{\mathrm{a}}$ & $1000 \pm 16^{\mathrm{a}}$ & $0,35 \pm 0,02^{\mathrm{c}}$ \\
\hline FM4 & $5,80 \pm 0,32^{\mathrm{c}}$ & $33,74 \pm 1,58^{\mathrm{a}}$ & $75,80 \pm 1,17^{\mathrm{ns}}$ & $2203 \pm 8^{c}$ & $5,74 \pm 0,09^{\mathrm{b}}$ & $1825 \pm 44^{\mathrm{a}}$ & $378 \pm 52^{\mathrm{b}}$ & $2635 \pm 26^{\mathrm{c}}$ & $810 \pm 18^{c}$ & $0,29 \pm 0,01^{\mathrm{d}}$ \\
\hline FM5 & $5,65 \pm 0,16^{\mathrm{c}}$ & $23,51 \pm 0,49^{b}$ & $75,00 \pm 0,07^{\mathrm{ns}}$ & $2248 \pm 2^{\mathrm{c}}$ & $5,47 \pm 0,09^{\mathrm{b}}$ & $1819 \pm 34^{\mathrm{a}}$ & $429 \pm 32^{b}$ & $2648 \pm 31^{\mathrm{c}}$ & $829 \pm 3,5^{\mathrm{c}}$ & $0,32 \pm 0,02^{\mathrm{d}}$ \\
\hline FM6 & $7,26 \pm 0,07^{\mathrm{a}}$ & $12,51 \pm 0,80^{\mathrm{c}}$ & $77,80 \pm 0,49^{\text {ns }}$ & $2403 \pm 33^{\mathrm{a}}$ & $5,97 \pm 0,09^{\mathrm{b}}$ & $1856 \pm 33^{\mathrm{a}}$ & $548 \pm 10^{\mathrm{a}}$ & $2768 \pm 7,5^{\mathrm{b}}$ & $912 \pm 25^{\mathrm{b}}$ & $0,42 \pm 0,03^{\mathrm{b}}$ \\
\hline FM7 & $5,41 \pm 0,44^{\mathrm{c}}$ & $12,44 \pm 0,02^{\mathrm{c}}$ & $77,10 \pm 0,60^{\mathrm{ns}}$ & $1313 \pm 11^{\mathrm{e}}$ & $4,53 \pm 0,01^{\mathrm{c}}$ & $774 \pm 2$ & $539 \pm 10$ & $1213 \pm 8^{\mathrm{g}}$ & $440 \pm 6,5^{\mathrm{e}}$ & $0,41 \pm 0,01^{\mathrm{b}}$ \\
\hline FM8 & $5,06 \pm 0,22^{\mathrm{c}}$ & $10,72 \pm 0,44^{\mathrm{c}}$ & $76,20 \pm 0,60^{\mathrm{ns}}$ & $1314 \pm 20^{\mathrm{e}}$ & $5,60 \pm 0,01^{\mathrm{b}}$ & $1045 \pm 29^{c}$ & $270 \pm 27^{\mathrm{c}}$ & $1682 \pm 9^{f}$ & $638 \pm 20^{\mathrm{d}}$ & $0,30 \pm 0,03^{\mathrm{d}}$ \\
\hline FM9 & $5,29 \pm 0,63^{c}$ & $6,69 \pm 0,08^{\mathrm{e}}$ & $77,10 \pm 0,42^{\mathrm{ns}}$ & $2323 \pm 25^{b}$ & $5,87 \pm 0,09^{\mathrm{b}}$ & $1866 \pm 31^{\mathrm{a}}$ & $457 \pm 6^{\mathrm{b}}$ & $2814 \pm 28^{b}$ & $948 \pm 3^{b}$ & $0,48 \pm 0,03^{\mathrm{a}}$ \\
\hline
\end{tabular}

As demais farinhas apresentaram diferença significativa em relação ao ISA, demonstrando que um estudo detalhado dos processos poderá contribuir para que o pequeno produtor possa obter farinhas com propriedades diferentes, caso ele faça ajustes nas condições técnicas de produção. Por exemplo, a FM4, que 
apresentou granulometria com menor retenção na peneira $2 \mathrm{~mm}$ (Tabela 1), foi a que teve o maior ISA, quando comparada com as demais.

A influência da granulometria na solubilidade já foi explicada por Brito et al. (2015), que verificaram que farinhas com granulometria fina apresentam maiores concentrações de carboidratos solúveis, devido à maior desestruturação dos tecidos vegetais, enquanto a farinha com maior granulometria apresenta menor concentração. Segundo os autores, maiores valores de ISA podem ser consequência do processamento das farinhas, as quais, ao serem submetidas a um tratamento térmico mais intenso durante a torração, apresentam uma alteração maior nos grânulos, devido à maior degradação das moléculas de amido em moléculas menores (dextrinas), que são mais solúveis em água.

IAA e ISA refletem nas caraterísticas da farinha para uso direto nos produtos, como espessante sem uso de tratamento térmico, como o hábito alimentar de se misturar farinhas com leite, café, açaí, entre outros. As propriedades viscoamilográficas (Tabela 3) indicam o comportamento das mesmas durante os processos de aquecimento em água e resfriamento, quando utilizadas na produção de sopas, caldos, pirão etc.

Os perfis viscoamilográficos das farinhas de mandioca FM1 a FM6 e FM9 apresentaram curvas de viscosidade obtidas durante aquecimento e resfriamento com os maiores valores e similares entre si. Assim, não seria possível distingui-las por esta análise, entretanto foram distintas das FM7 e FM8, que apresentaram os menores parâmetros, como pode ser observado na Tabela 3 e na Figura 3.

A presença de amido cru foi visível nas curvas viscoamilográficas, sendo que as farinhas do grupo 1 (FM1 a FM6 e FM9) apresentaram curvas de gelatinização com maiores valores do que as do grupo 2 (FM7 e FM8). Este fato contribui para explicar as diferenças de digestibilidade encontradas para farinhas de mandioca, conforme resultados verificados por Brito et al. (2015), e reforça a indicação de consumo direto da farinha para obter saciedade, pois apresenta carboidratos de rápida digestão (devido aos valores de ISA, que indicam presença de dextrinas), amidos parcialmente gelatinizados (menores valores de viscosidade máxima) e amidos crus (curvas típicas de gelatinização e retrogradação do amido) e fibras alimentares. Porém, se usadas como espessantes e forem cozidas juntas, terão carboidratos rapidamente digeríveis, pois todo o amido será facilmente gelatinizado.

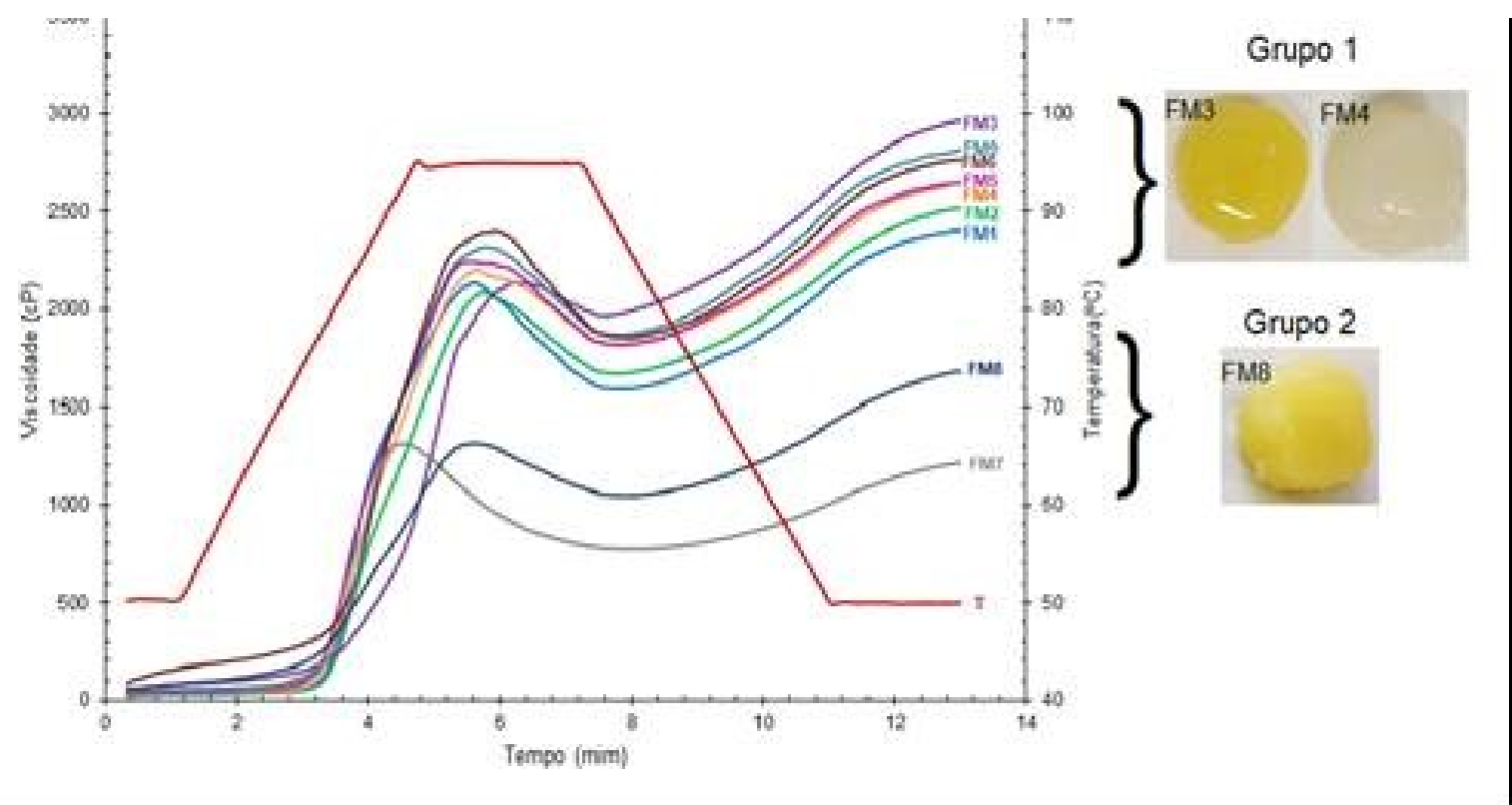

Figura 3. Propriedades de pastas das farinhas de mandioca e imagem dos géis obtidos representativos do grupo 1 (maiores viscosidades) e do grupo 2 (menores viscosidades). 
Verificou-se que não houve diferença significativa entre as farinhas de mandioca em relação à temperatura de pasta (Tp), indicando que todas poderão ser gelatinizadas na faixa de $75{ }^{\circ} \mathrm{C}$ a $80^{\circ} \mathrm{C}$, que corresponde ao amido de mandioca puro, que foi analisado por Oliveira (2011).

As FM6 e FM9 apresentaram maiores viscosidades máximas a $95^{\circ} \mathrm{C}(\mathrm{VM})$, indicando que seria necessário maior aporte de energia durante a gelatinização para que ocorresse o aquecimento e a agitação da pasta, sendo estas assim indicadas para produção de alimentos que requerem maior viscosidade. Entretanto, foram menores que os valores encontrados por Oliveira (2011) para amido cru (4.417,00 a 3.527,50 cP). Este fato indica que as FM analisadas apresentaram modificações no amido, devido ao tratamento térmico utilizado durante o processamento, pois houve diminuição da viscosidade de pasta.

Verificou-se que houve diferenças entre as características das farinhas em relação à retrogradação $(\mathrm{R})$, com valores menores dos que os apresentados por Oliveira (2011) (1.036,00 a 1.360,00 cP). As FM7 e FM8 apresentaram menor tendência à retrogradação, sendo indicadas para melhorar a qualidade de pudins $\mathrm{e}$ molhos, e regionalmente em Belém-PA, Brasil, como a mais indicada na elaboração de pirão. É importante ressaltar que, segundo Denardin \& Silva (2009), a tendência à retrogradação é um parâmetro que auxilia na estimativa da estabilidade do gel de amido na estocagem e na perda de água (sinérese) de algumas sobremesas.

Verificou-se que as farinhas FM6 e FM9, que apresentaram maiores viscosidades finais a $50{ }^{\circ} \mathrm{C}(\mathrm{Vf})$, também apresentaram maior força do gel (Tabela 3), que foi analisada após 24 horas de repouso, em refrigeração.

As pastas formadas pelas farinhas de mandioca foram claras, translúcidas, com textura longa, mas não firmes (Figura 3), confirmando as características indicadas por Cereda \& Vilpoux (2003). As farinhas FM4, FM5, FM6 apresentaram géis de cor creme e as outras amostras apresentaram géis de cor amarela (Figura 3).

\section{Conclusão}

As farinhas de mandioca puderam ser diferenciadas em relação à cor e, principalmente, à granulometria, parâmetros que afetaram as suas propriedades tecnológicas, mas com potencial para serem utilizadas em panificação. Estas variações nas características permitiram verificar que são necessárias modificações no processamento artesanal das farinhas, como tempo e temperaturas durante torração, para padronização da qualidade das farinhas utilizadas como ingrediente alimentício sem glúten.

\section{Agradecimentos}

Os autores agradecem à Coordenação de Aperfeiçoamento de Pessoal de Nível Superior (CAPES) - código financeiro 001; ao CNPq, pela bolsa de doutorado e pela taxa de bancada (Processos 140807/2016-0 e 142287/2019-9) de Elisa Cristina Andrade Neves, e ao CNPq e à FAPESP (Processo 2016/12184-7), pelas bolsas de Iniciação Científica de Gustavo Nascimento Costa.

\section{Referências}

Álvares, V. S., Miqueloni, D. P., \& Negreiros, J. R. S. (2016). Variabilidade físico-química da farinha de mandioca do Território da Cidadania do Vale do Juruá, Acre. Revista Ceres, 63(2), 113-121. http://dx.doi.org/10.1590/0034-737X201663020001

Anderson, R. A., Conway, H. F., Pfeifer, V. F., \& Griffin Junior, E. L. (1969). Gelatinization of corn grits by roll and extrusion cooking. Cereal Science Today, 14(1), 372-376.

Association of Official Analytical Chemists - AOAC. (2005). Official methods of analysis of the AOAC International. Arlington: AOAC.

Aryee, F. N. A., Oduro, I., Ellis, W. O., \& Afuakwa, J. J. (2006). The physicochemical properties of flour samples from the roots of 31 varieties of cassava. Food Control, 17(11), 916-922. http://dx.doi.org/10.1016/j.foodcont.2005.06.013

Bezerra, V. S. (2006). Farinhas de mandioca seca e mista (Coleção Agroindústria familiar, 44 p.). Brasília: Embrapa Informação Tecnológica. 
Brasil. Ministério da Agricultura, Pecuária e Abastecimento. (2011, novembro 7). Regulamento Técnico da Farinha de Mandioca (Instrução Normativa $n^{\circ}$ 52, de 7 de novembro de 2011). Diário Oficial [da] República Federativa do Brasil, Brasília. Retrieved in 2019, February 09, from

http://sistemasweb.agricultura.gov.br/sislegis/action/detalhaAto.do?method=visualizarAtoPortalMapa\&chave=497488882

Brimer, L., \& Rosling, H. (1993). Microdiffusion method with solid-state detection of cyanogenic glycosides from cassava in human urine. Food and Chemical Toxicology, 31(8), 599-603. PMid:8349206. http://dx.doi.org/10.1016/0278-6915(93)90210-P

Brito, V. H. S., Silva, E. C., \& Cereda, M. P. (2015). Digestibilidade do amido in vitro e valor calórico dos grupos de farinhas de mandioca brasileiras. Brazilian Journal of Food Technology, 18(3), 185-191. http://dx.doi.org/10.1590/1981-6723.2714

Cereda, M. P., \& Vilpoux, O. F. (2003). Tecnologia, usos e potencialidades de tuberosas amiláceas latino americanas (Culturas de tuberosas amiláceas latino americanas, propriedades gerais do amido, v. 3). São Paulo: Fundação Cargill.

Chisté, R. C., Cohen, K. O., Mathias, E. A., \& Oliveira, S. S. (2010). Quantificação de cianeto total nas etapas de processamento das farinhas de mandioca dos grupos seca e d'água. Acta Amazonica, 40(1), 221-226. http://dx.doi.org/10.1590/S0044-59672010000100028

Chisté, R. C., Cohen, K. O., Mathias, E. A., \& Ramoa Júnior, A. G. A. (2006). Qualidade da farinha de mandioca do grupo seca. Food Science and Technology, 26(4), 861-864. http://dx.doi.org/10.1590/S0101-20612006000400023

Cohen, K. O., Oliveira, S. S., \& Chisté, R. C. (2007). Quantificação de teores de compostos cianogênicos totais em produtos elaborados com raízes de mandioca. Belém: Embrapa Amazônia Oriental.

Dias, L. T., \& Leonel, M. (2006). Caracterização físico-química de farinhas de mandioca de diferentes localidades do Brasil. Ciência e Agrotecnologia, 30(4), 692-700. http://dx.doi.org/10.1590/\$1413-70542006000400015

Denardin, C. C., \& Silva, L. P. (2009). Estrutura dos grânulos de amido e sua relação com propriedades físico-químicas. Ciência Rural, 39(3), 945-954. http://dx.doi.org/10.1590/S0103-84782009005000003

Eleazu, C. O., Amajor, J. U., Ikpeama, A. I., \& Awa, E. (2011). Studies on the nutrient composition, antioxidant activities, functional properties am microbial load of the flours of 10 elite cassava (Manihot esculenta) varieties. Asian Journal of Clinical Nutrition, 3(1), 33-39. http://dx.doi.org/10.3923/ajcn.2011.33.39

Ferreira, D. F. (2008). SISVAR: um programa para análises e ensino de estatística. Revista Científica Symposium, 6(2), 36-41.

Franck, H., Christian, M., Noël, A., Brigitte, P., Joseph, H. D., Cornet, D., \& Mathurin, N. D. (2011). Effects of cultivar and harvesting conditions on the texture and taste of boiled cassava roots. Food Chemistry, 126(1), 127-133. http://dx.doi.org/10.1016/j.foodchem.2010.10.088

Instituto Brasileiro de Geografia e Estatística - IBGE. (2019). Levantamento sistemático da produção agrícola. Rio de Janeiro: IBGE. Retrieved in 2019, February 09, from: https://sidra.ibge.gov.br/tabela/1618\#resultado

Jay, J. M. (2009). Microbiologia de alimentos (6. ed.). Porto Alegre: The Art of Medication.

Oliveira, D. C. (2011). Caracterização e potencial tecnológico de amidos de diferentes cultivares de mandioca (Manihot esculenta Crantz) (Dissertação de mestrado). Universidade Federal de Santa Catarina, Florianópolis.

Oliveira, F. A. D., \& Oliveira, F. C. (2010). Toxicologia experimental de alimentos. Porto Alegre: Sulina.

Silva, A. C. M. S., Pinho, L. P., Sousa, L. S., Moura, L. E., Souza, C. O., \& Druzian, J. I. (2015). Classificação, identidade e matérias estranhas de farinha de mandioca Copioba: conformidade com a legislação brasileira e contribuição a indicação geográfica. Caderno de Prospecção, 8(1), 192-202. http://dx.doi.org/10.9771/S.CPROSP.2015.001.022

Tabela Brasileira de Composição de Alimentos - TACO (2011). 4. ed. revisada e ampliada. Campinas: NEPA/UNICAMP.

Funding: Coordenação de Aperfeiçoamento de Pessoal de Nível Superior (CAPES)-código 001, CNPq (Processos 140807/2016-0 e 142287/2019-9), CNPq / FAPESP (Processo 2016/12184-7). 